\title{
In-situ synchrotron diffraction study of precipitations in liquid jet
}

Ahmed S. A. Mohammed $^{1}$, Antonio Cervellino ${ }^{1}$, Andrea Testino ${ }^{2}$, Agnese Carino ${ }^{2}$

${ }^{1}$ Swiss Light Source, Paul Scherrer Institut, Ch-5232 Villigen, Switzerland, ${ }^{2}$ Energy and Environment Research Division (ENE), Paul Scherrer Institut (PSI), Ch-5232 Villigen, Switzerland

E-mail: ahmed.mohammed@psi.ch

Calcium carbonate, one of the most studied biominerals, has major applications across a broad spectrum of technologies. In order to investigate the crystallization kinetics, in-situ wide-angle (WAXS) and small-angle (SAXS) synchrotron X-ray scattering experiments are being performed at the MS-X04AS Beamline of the SLS synchrotron at the PSI, Villigen, Switzerland. [1] In particular, the SAXS signal is important for detecting aggregates and their size, independently of their atomic structure, while the WAXS will enable us to distinguish between amorphous clumps and crystalline NPs. The feasibility of the WAXS data collection has been established in recent tests, while a SAXS experiment has been performed in situ on a horizontal liquid microjet. This was generated using a nozzle connected to a mixer. Four HPLC pumps were delivering solutions in order to obtain the desirable $\mathrm{pH}$ and saturation level of the system. The liquid was collected in a catcher where $\mathrm{T}$ and $\mathrm{pH}$ of the solution, under stirring, were monitored on line. After micro-jet optimization (pulsation damping, liquid jet diameter, solution composition, time delay between mixing point and liquid, X-ray beam focusing), measurements were carried out with stainless steel nozzles of $125 \mu \mathrm{m}$ and $250 \mu \mathrm{m}$ and with delay times of $0,1,60 \mathrm{~s}$. The SAXS data (figure 1 shows a representative example of the data), collected using a Mythen II detector [2], revealed very clearly pre-nucleation spherical amorphous clusters of 20-30 nm size. Correlations between supersaturations, delay time after mixing, particle size, and concentrations are analyzed and discussed [3]. The data are corrected from air and sodium carbonate solution background.

[1] P. R. Willmott et al., J. Synchrotron Rad. (2013) 20, 667-682

[2] A. Bergamaschi et al., J. Synchrotron Rad. (2010). 17, 653-668

[3] Ahmed S. A. Mohammed et al., in preparation, (2017)

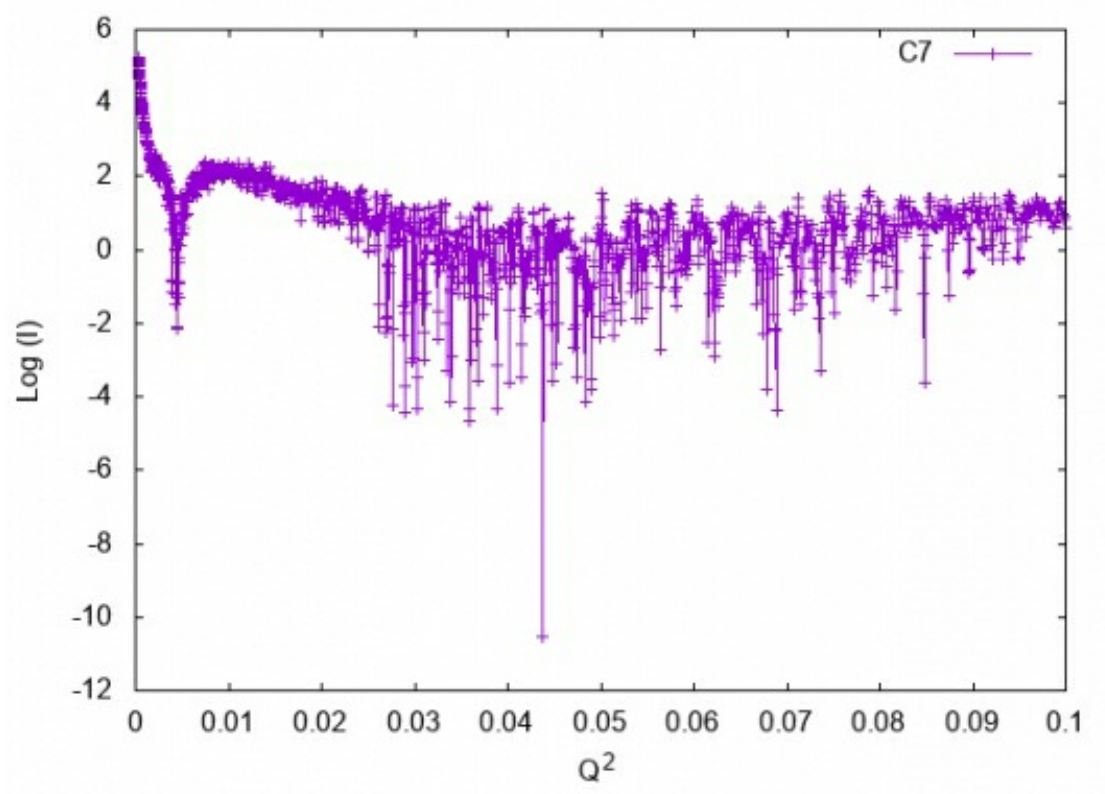

Keywords: SAXS, liquid Jet, Precipitation 